\title{
A Method for the Measurement of Photons Number and Squeezing Parameter in a Quantum Cavity
}

\author{
Ghasem Naeimi, Siamak Khademi, and Ozra Heibati \\ Department of Physics, University of Zanjan, P.O. Box 45196-313, Zanjan, Iran \\ Correspondence should be addressed to Siamak Khademi; khademi@znu.ac.ir
}

Received 20 September 2013; Accepted 7 November 2013

Academic Editors: Y. S. Kivshar, X. Shu, and Y. Tsuji

Copyright (c) 2013 Ghasem Naeimi et al. This is an open access article distributed under the Creative Commons Attribution License, which permits unrestricted use, distribution, and reproduction in any medium, provided the original work is properly cited.

\begin{abstract}
Measurement of photons number in a quantum cavity is very difficult and the photons number is changed after each measurement. Recently, many efforts have been done for the nondemolition measurement methods. Haroche et al. succeed in recognizing existence or nonexistence of one photon in a quantum cavity. In this paper, we employ their experimental setup for a quantum nondemolition measurement and pump a coherent state in their quantum cavity. In this case, we could detect more photons in the quantum cavity by a measurement of a displaced Wigner function. It is also shown that the measurement of more than one photon is possible by the Haroche method by measuring just one point of displaced Wigner function. Furthermore, if the cavity field is filled by a superposition of two number states, the average number of photons within the cavity would be measurable. We show that their setup is also suitable to apply for the measurement of the squeezing parameter for the squeezed state of photons number in the quantum cavity successfully.
\end{abstract}

\section{Introduction}

The formulation of quantum mechanics in phase space was proposed by Wigner [1]. This formulation is very useful in various fields of physics including quantum mechanics [2, $3]$, quantum optics [4-6], and condensate matter $[7,8]$. The physical concepts are extractable from Wigner function. Wigner function may take negative value for a quantum state. The existence of negative or interference of Wigner function is a nonclassicality indicator for quantum systems [9-11]. On the other hand, Wigner function is a measurable quantity. Many authors introduced methods to measure Wigner function for trapped ions [12], photonic number states in quantum cavity [13-15], Schrodinger cat state, and coherent state [16]. Bertet et al. measure a complete Wigner function for the vacuum and a single photon state [17]. Lutterbach and Davidovich presented a method to measure the Wigner distribution function of photonic state in a quantum cavity field $[18,19]$. They used an experimental ingenious setup which was made by one high Q-factor and two low Q-factor cavities.

Nogues et al. (members of Haroche group) measured the Wigner distribution functions of electromagnetic fields in a cavity with the number states $n=0$ and $n=1$ at origin of phase space [20]. The Wigner distribution function at the origin of phase space is positive for $n=0$ and negative for $n=1$. Therefore, the sign of measured Wigner distribution function, itself, gives us the number of photons in the cavity and its value is not important [20]. So, if there are more than one photon, it would not be possible to recognize the number of photons. In this paper we used the Haroche method to measure the larger number of photons by measuring just one point of the displaced Wigner distribution function in a quantum cavity. We use an experimental setup for the measurement of displaced Wigner function proposed by Deléglise et al. [16] (Haroche group). It is shown that their experimental setup is useful for the measurement of number of photons, even for $n>1$. This method is also suitable to measure the average number of superpositions of two number states. The development of method to an arbitrary superposition of states needs more measurement for many more points of Wigner function which is not discussed in this paper. Furthermore this method is also applied to measure the squeezing parameter for squeezed number state of photons.

In the next section, the Wigner distribution function is calculated for four values of $n$ and their plot in phase space is illustrated. It would be shown that Wigner functions at 
the origin of phase space have positive value for the even and negative value for the odd number of photons. So, by measuring the Wigner function at the origin we only find whether the number of photons is even or odd. The value of the displaced Wigner function depends on the photons number. We find a point in phase space in which Wigner functions have different values for different photons number. Therefore we determine the photons number (or the average of photons number) by measuring the displaced Wigner function. In this section Lutterbach and Davidovich method is developed for displaced Wigner function and its experimental setup will be introduced. The measurement of displaced Wigner function in a quantum cavity is compared with the value of the Wigner function of different number states. Then the number of photons is obtained. In Section 3 a quantum cavity field is set to be in a superposition of two number states. In this case the average number of photons in the cavity is measured by calculating the Wigner function in the quantum cavity field by comparing the result with the Wigner function measured by the Latterbach and Davidovich proposed experimental setup. Section 4 is devoted to the measurement of squeezing parameter for the squeezed number states in a quantum cavity. The displaced Wigner function is measured for the squeezed field by developed Lutterbach and Davidovich experimental setup. The squeezed parameter is measurable by calculating the displaced Wigner function, for any value of $n$. Finally, the last section is devoted to the conclusions.

\section{Measuring Number of Photons in a Quantum Cavity}

2.1. Calculating the Wigner Function. The Wigner function for a number state $|n\rangle$ is given by [21]

$$
W(\alpha)=2 \operatorname{Tr}\left(\widehat{D}^{-1}(\alpha) \widehat{\rho} \widehat{D}(\alpha) \widehat{P}\right),
$$

where $\widehat{\rho}=|n\rangle\langle n|$ is the density operator, $\widehat{P}=e^{i \pi \widehat{a}^{\dagger} \widehat{a}}$ is the parity operator, and $\widehat{D}(\alpha)=e^{\left(\alpha \hat{a}^{\dagger}-\alpha^{*} \widehat{a}\right)}$ is the displacement operator in phase space $[21,22]$. The operation of the parity and displacement operators on $|n\rangle$ is given by [21]

$$
\begin{gathered}
\widehat{P}|n\rangle=e^{i \pi \widehat{a}^{\dagger} \widehat{a}}|n\rangle=(-1)^{n}|n\rangle, \\
\widehat{D}(\alpha)|n\rangle=e^{\left(\alpha \widehat{a}^{\dagger}-\alpha^{*} \widehat{a}\right)}|n\rangle=|n, \alpha\rangle,
\end{gathered}
$$

where $|n, \alpha\rangle$ is the displaced number state [21]. Equation (1) can be written as [21]

$$
W(\alpha)=2 \operatorname{Tr}(\widehat{\rho} \widehat{D}(2 \alpha) \widehat{P})=2 \sum_{m=0}^{\infty}\langle m|(\widehat{\rho} \widehat{D}(2 \alpha) \widehat{P})| m\rangle,
$$

where $|m\rangle$ is a number state. By (2) and (3) the Wigner function is obtained as follows:

$$
\begin{aligned}
W_{n}(\alpha) & =2 \sum_{m=0}^{\infty}(-1)^{n} \delta_{n m}\langle n \mid m, 2 \alpha\rangle \\
& =2(-1)^{n} e^{-2|\alpha|^{2}} L_{n}\left(4|\alpha|^{2}\right) .
\end{aligned}
$$

The value of $\langle n \mid m, 2 \alpha\rangle$ for $n<m$ and $n \geq m$ is written versus Laguerre polynomials as

$$
\begin{gathered}
\langle n \mid m, 2 \alpha\rangle=e^{-2|\alpha|^{2}}\left(-\alpha^{*}\right)^{m-n} \sqrt{\frac{n !}{m !}} L_{n}^{(m-n)}\left(4|\alpha|^{2}\right), \\
\langle n \mid m, 2 \alpha\rangle=e^{-2|\alpha|^{2}}(\alpha)^{n-m} \sqrt{\frac{m !}{n !}} L_{m}^{(n-m)}\left(4|\alpha|^{2}\right),
\end{gathered}
$$

respectively. By the above relations the Laguerre polynomials for $n=m$ are given by

$$
L_{n}(x)=\frac{e^{x}}{n !} \frac{d^{n}}{d x^{n}}\left(e^{-x} x^{n}\right),
$$

where $x=4|\alpha|^{2}$. Let us consider a cavity with $n$ photons. The exact value of $n$ is not definite, but suppose there is a few number of photons, for example, between 0 and 3. From (4) to (6) the Wigner function is calculated for number states as

$$
\begin{gathered}
W_{0}(\alpha)=2 e^{-2|\alpha|^{2}}, \\
W_{1}(\alpha)=-2 e^{-2|\alpha|^{2}}\left(1-4|\alpha|^{2}\right), \\
W_{2}(\alpha)=\frac{2 e^{-2|\alpha|^{2}}}{2 !}\left(16|\alpha|^{4}-16|\alpha|^{2}+2\right), \\
W_{3}(\alpha)=\frac{-2 e^{-2|\alpha|^{2}}}{3 !}\left(-64|\alpha|^{6}+144|\alpha|^{4}-7|\alpha|^{2}+6\right) .
\end{gathered}
$$

In Figure $1, \operatorname{Re}(\alpha)$ and $\operatorname{Im}(\alpha)$ are the axes of phase space. The cross section of the Wigner functions for the number states is plotted in terms of $\operatorname{Re}(\alpha)$ for $\operatorname{Im}(\alpha)=0$. These Wigner functions have just two values in the origin; for even number states they are positive, while for odd number states they are negative. Thus, the value of the Wigner function at the origin is not sufficient to specify the number of photons in the quantum cavity. For other points of phase space these values are not the same for different states of photon numbers. One can apply this feature to specify the number of photons in the cavity.

To select a point in the phase space in which the Wigner function and consequently the number of photons are going to be measured, we note that the values of the Wigner function for each $n$ should have the maximum difference as much as possible. In order to have exact and significant measurement the discrepancy should be much more than measuring errors. In Figure 2 the value of Wigner functions versus the number of photons has been plotted for $\operatorname{Re}(\alpha)=$ 0.5 .

2.2. The Measurement of Photons Number. In this section we apply the Lutterbach and Davidovich method for measuring the Wigner function of the electromagnetic field in the cavity, where the number of photons is one of the $n$ values (e.g. $n=$ $0,1,2$, and 3 ).

As shown in Figure 3, the experimental setup is made of 3 cavities. The quantum field in cavity $C$ is determined by an eigenstate of number states, but $R_{1}$ and $R_{2}$ cavities contain 


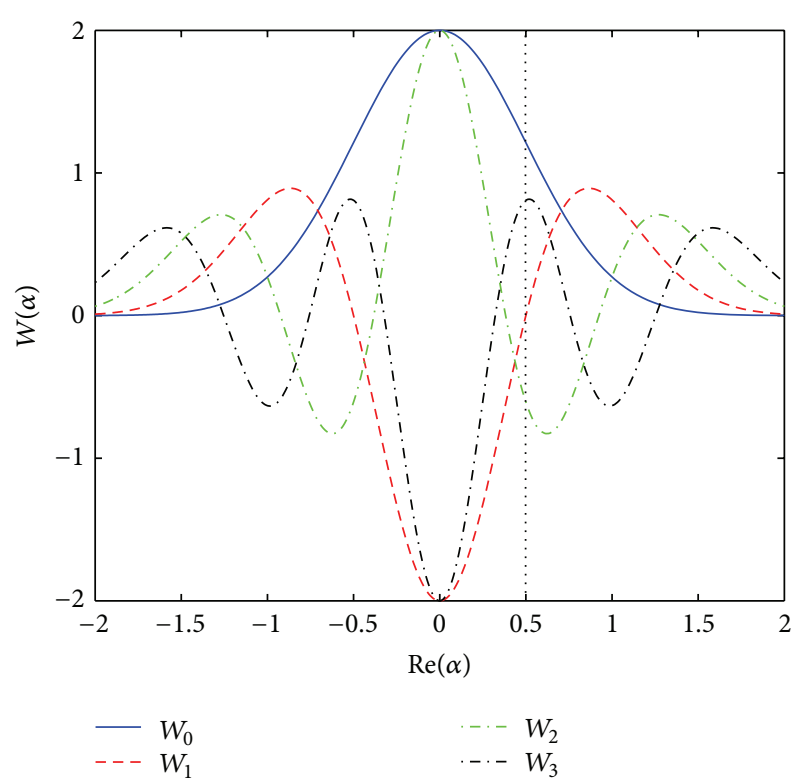

FIgure 1: The Wigner function is plotted versus $\operatorname{Re}(\alpha)$ for $n=$ $0,1,2,3 . W_{0}, W_{1}, W_{2}$, and $W_{3}$ are the Wigner distribution functions for $n=0,1,2,3$, respectively. The Wigner distribution functions at the origin have only two values for both the even and odd numbers of photons. Outside the origin, for example, at $\operatorname{Re}(\alpha)=0.5$, values of the Wigner distribution functions are not the same for different number of photons.

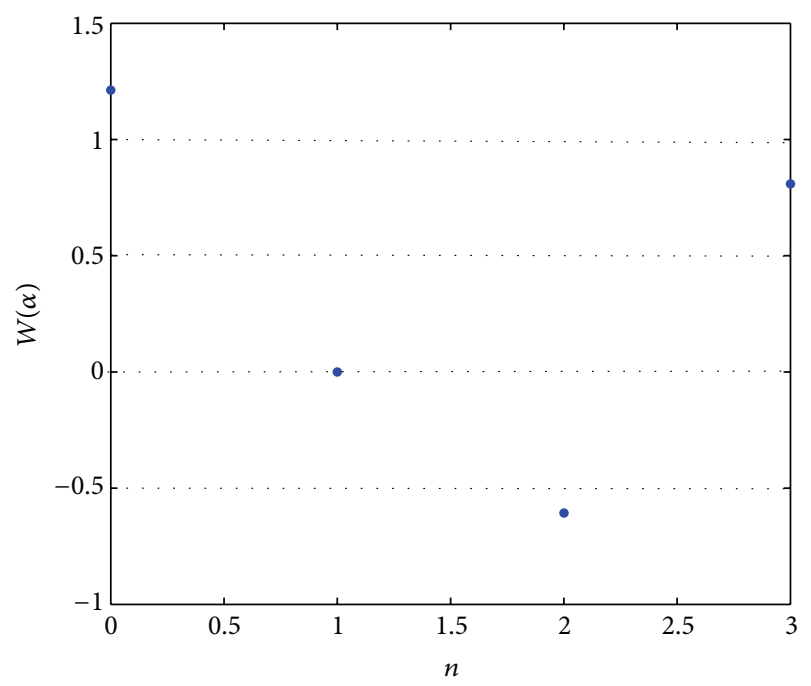

Figure 2: The values of Wigner function for the photon numbers (0 to 3 ) for $\operatorname{Re}(\alpha)=0.5$. It is necessary to reduce the experimental error, in order to avoid the overlap of the errors in neighbor points.

classical fields. Each cavity is a Fabry-Perot resonator which is made of two spherical super conductor mirrors [20].

In this experiment, a beam of Rydberg rubidium atoms pass through the cavities and interact with their electromagnetic fields. If one of the electrons of the atoms (usually the valence electron) is excited and jumps to a level with a higher quantum number, the atom would settle in a Rydberg state.

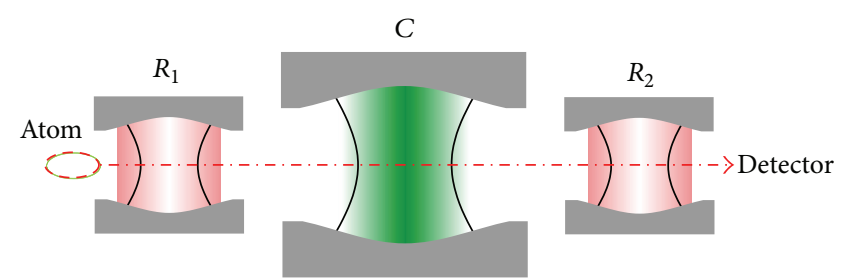

FIGURE 3: The experimental scheme for measuring the Wigner functions in the electromagnetic field in cavity $C$, which leads us to measure the number of photons. The cavities $R_{1}$ and $R_{2}$ contain the classical electromagnetic fields.

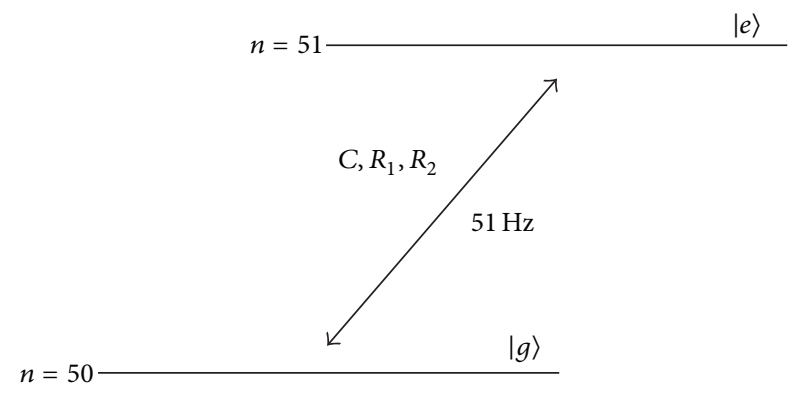

FIGURE 4: The Rydberg levels for the rubidium atom which interacts with all cavities in Figure 3.

Here, two Rydberg levels with quantum numbers, 50 and 51 , are investigated and their states denoted by $|g\rangle$ and $|e\rangle$, respectively (see Figure 4). The frequency of the field of each cavity is set to be close to the transition frequency between these two levels. So, the rubidium atom interacts with the field as a two-level atom. The interaction of the rubidium atom with the field of $R_{1}$ and $R_{2}$ cavities is performed in a resonant state. In the cavity $R_{1}$, the atom in the $|e\rangle$ state emits a photon during the interaction with the field and jumps to $|g\rangle$ state. The atom may absorb this photon and come back to the $|e\rangle$ state again. This happens over and over and the atom oscillates between the two levels. These oscillations are called Rabi oscillations. The Rabi frequency is denoted by $\Omega_{R}$. In general, during interaction, the state of atom evolves to a superposition of states [23] as follows:

$$
|e\rangle \stackrel{R_{1}}{\longrightarrow} \cos \left(\frac{\Omega_{R} t}{2}\right)|e\rangle+\sin \left(\frac{\Omega_{R} t}{2}\right)|g\rangle,
$$

where $\Omega_{R} t$ is a phase which is obtained by the atom while passing through the cavity $R_{1}$. Here we consider $\Omega_{R} t=\pi / 2$; then

$$
|e\rangle \stackrel{R_{1}}{\longrightarrow} \frac{1}{\sqrt{2}}(|e\rangle+|g\rangle) \text {. }
$$

In the cavity $C$, by applying a uniform electric field, the Stark effect causes a small difference between the cavity field frequency $\omega_{f}$ and the two-level atom transition frequency $\omega_{a}=\left(E_{e}-E_{g}\right) / \hbar$, which is called detuning $\delta=\omega_{f}-\omega_{a}$. In a nonresonance interaction, there is no transition between the 
atomic levels. Such nonresonance interaction applies a phase shift to the atomic state. This phase shift is given by [23]

$$
\begin{gathered}
e^{-i \widehat{H}_{\text {eff }} / \hbar}|e\rangle|n, \alpha\rangle=e^{-i \varphi(\widehat{n}+1) t}|e\rangle|n, \alpha\rangle, \\
e^{-i \widehat{H}_{\text {eff }} / \hbar}|g\rangle|n, \alpha\rangle=e^{-i \varphi \widehat{n} t}|g\rangle|n, \alpha\rangle,
\end{gathered}
$$

for $|e\rangle$ and $|g\rangle$ states, respectively. In these relations, $\left.\widehat{H}_{\mathrm{eff}}=\hbar \varphi\left[\left(1+\hat{a}^{\dagger} \widehat{a}\right)|e\rangle\left\langle e\left|-\hat{a}^{\dagger} \widehat{a}\right| g\right\rangle\langle g|\right)\right]$ is the interaction Hamiltonian, $\varphi=\lambda^{2} / \delta=d^{2} \varepsilon^{2} / \hbar^{2} \delta$ depends on $\varepsilon=-\left(\hbar \omega / \varepsilon_{0} V\right)^{1 / 2} \sin k z$ which is equal to the electric field amplitude, and $\widehat{E}=\left(\hbar \omega / \varepsilon_{\mathrm{o}} V\right)^{1 / 2}\left(\widehat{a}^{\dagger}+\widehat{a}\right) \sin k z$ [23]. In order to measure the displaced Wigner function a coherent electromagnetic field is pumped into the cavity $C$. The effect of this coherent field on the number state of photon is a displacement which is shown by $|n, \alpha\rangle=\widehat{D}(\alpha)|n\rangle$. The state of the atom outgoing the cavity $C$ is given by

$$
\stackrel{C}{\rightarrow} \frac{1}{\sqrt{2}}\left(e^{-i \varphi\left(\widehat{a}^{\dagger} \widehat{a}+1\right) t}|e\rangle+e^{-i \varphi \widehat{a}^{\dagger} \widehat{a} t}|g\rangle\right) .
$$

The relative phase between the cavities $R_{1}$ and $R_{2}$ is $\eta$ [18]. The atom makes also an interaction with a phase $\Omega_{R} t=\pi / 2$ in the cavity $R_{2}$ resonantly. Thus each part of states in (11) changes as follows:

$$
\begin{gathered}
|e\rangle \stackrel{R_{2}}{\longrightarrow} \frac{1}{\sqrt{2}}\left(|e\rangle+e^{i \eta}|g\rangle\right), \\
|g\rangle \stackrel{R_{2}}{\longrightarrow} \frac{1}{\sqrt{2}}\left(|g\rangle-e^{-i \eta}|e\rangle\right) .
\end{gathered}
$$

Therefore, after going out of the last cavity the total state of the system is given by

$$
\begin{aligned}
|\psi\rangle=\frac{1}{\sqrt{2}} & {\left[\left(e^{-i \varphi(\widehat{n}+1)}-e^{i(\varphi \widehat{n}-\eta)}\right)|e\rangle\right.} \\
& \left.+\left(e^{i(-\varphi((\widehat{n}+1)+\eta)}+e^{i \varphi \widehat{n}}\right)|g\rangle\right]|n, \alpha\rangle .
\end{aligned}
$$

By (13) the atom-field density matrix of the whole system is obtained as

$$
\begin{aligned}
\hat{\rho}_{\text {atom }+ \text { field }}=\frac{1}{4}[ & |e\rangle\langle e|\left(e^{-i \varphi(\widehat{n}+1)}-e^{i(\varphi \widehat{n}-\eta)}\right) \\
& \times \hat{\rho}^{\prime}\left(e^{+i \varphi(\widehat{n}+1)}-e^{-i(\varphi \widehat{n}-\eta)}\right) \\
& +|g\rangle\langle g|\left(e^{i(-\varphi(\widehat{n}+1)+\eta)}+e^{i \varphi \widehat{n}}\right) \\
& \times \hat{\rho}^{\prime}\left(e^{i(-\varphi(\widehat{n}+1)+\eta)}+e^{-i \varphi \widehat{n}}\right) \\
& + \text { nondiagonal terms of atomic state }]
\end{aligned}
$$

where $\widehat{\rho}^{\prime}=\widehat{D}(\alpha) \widehat{\rho} \widehat{D}^{-1}(\alpha)$. Then outgoing state of atom is detected by ionization detector in the $|e\rangle$ or $|g\rangle$ states. This experiment should be repeated many times and the probabilities of detecting the atoms in each $|e\rangle$ or $|g\rangle$ state are described with $P_{e}=N_{e} /\left(N_{e}+N_{g}\right)$ and $P_{g}=N_{g} /\left(N_{e}+N_{g}\right)$, respectively.
Here $N_{e}$ and $N_{g}$ are the numbers of detected atoms in $|e\rangle$ and $|g\rangle$ states, respectively. By (14), these probabilities and also the difference between them versus the Wigner function [18] are obtained as

$$
\Delta P=-\operatorname{Re}\left\{e^{i(\varphi-\eta)} \operatorname{Tr}\left[\widehat{D}(\alpha) \widehat{\rho} \widehat{D}^{-1}(\alpha) e^{2 i \varphi \widehat{a}^{\dagger} \widehat{a}}\right]\right\}
$$

where $\Delta P=P_{e}-P_{g}$. If we set $\varphi=-\eta=\pi / 2$, then $\Delta P$ would be proportional to a displaced Wigner function:

$$
\Delta P=P_{e}-P_{g}=\frac{W(-\alpha)}{2}
$$

The number of photons in the cavity $C$ is obtained by a comparison between the measured Wigner function at the point $\alpha,(16)$, and the value of the displaced Wigner functions for $n=0,1,2,3$. We can develop this method to measure a large number of photons, although the accuracy of our measurement is our main limitation.

\section{Measuring the Average Number of Photons in a Quantum Cavity}

In the previous experimental setup the cavity $C$ was in a number state $|n\rangle$ and the proposed experiment reveals integer photons number within the cavity. In this section we show if cavity $C$ can be in a superposition state; the average of photons number is also measurable by the same experimental method. This method is applied for different superpositions of number states. For example, consider the field of quantum cavity $C$ to be in a superposition state of $|0\rangle$ and $|1\rangle$ :

$$
\left|\psi^{\prime}\right\rangle=\sqrt{1-c^{2}}|0\rangle+c|1\rangle
$$

where $c=|c| \exp i \theta$. The average number of photons is independent of the phase $\theta$ :

$$
\bar{n}=\left\langle\psi^{\prime}|n| \psi^{\prime}\right\rangle=c^{2}
$$

In the next subsection we show that the measurement of the displaced Wigner function for a superposition of states gives us the average photon numbers and the phase $\theta$.

3.1. Calculating the Wigner Function for a Superposition of Number States. Suppose the cavity field is given by a superposition state $\left|\psi^{\prime}\right\rangle=\sqrt{1-c^{2}}|0\rangle+c|1\rangle$. By substituting the density operator $\hat{\rho}=\left|\psi^{\prime}\right\rangle\left\langle\psi^{\prime}\right|$ in (3) the Wigner function for the field in the cavity $C$ is obtained as

$$
\begin{aligned}
& W(\alpha)=2\left[\left(1-|c|^{2}\right)\langle 0 \mid 0,2 \alpha\rangle+\sqrt{1-c^{2}}\right. \\
& \quad \times c\left(e^{-i \theta}\langle 1 \mid 0,2 \alpha\rangle-e^{+i \theta}\langle 0 \mid 1,2 \alpha\rangle\right) \\
& \left.-|c|^{2}\langle 1 \mid 1,2 \alpha\rangle\right] .
\end{aligned}
$$




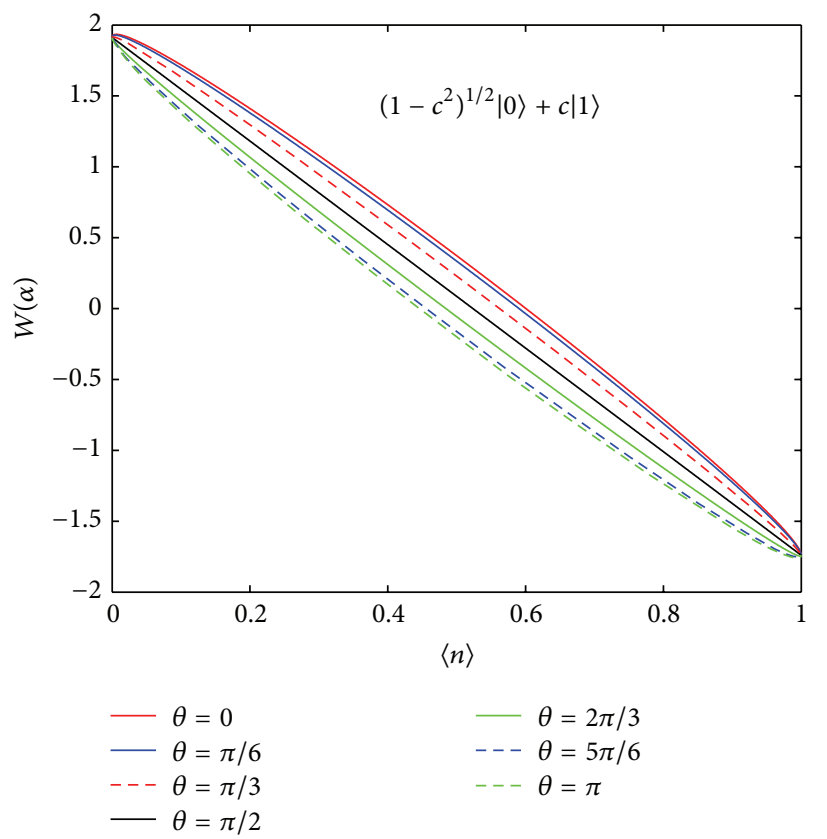

(a)

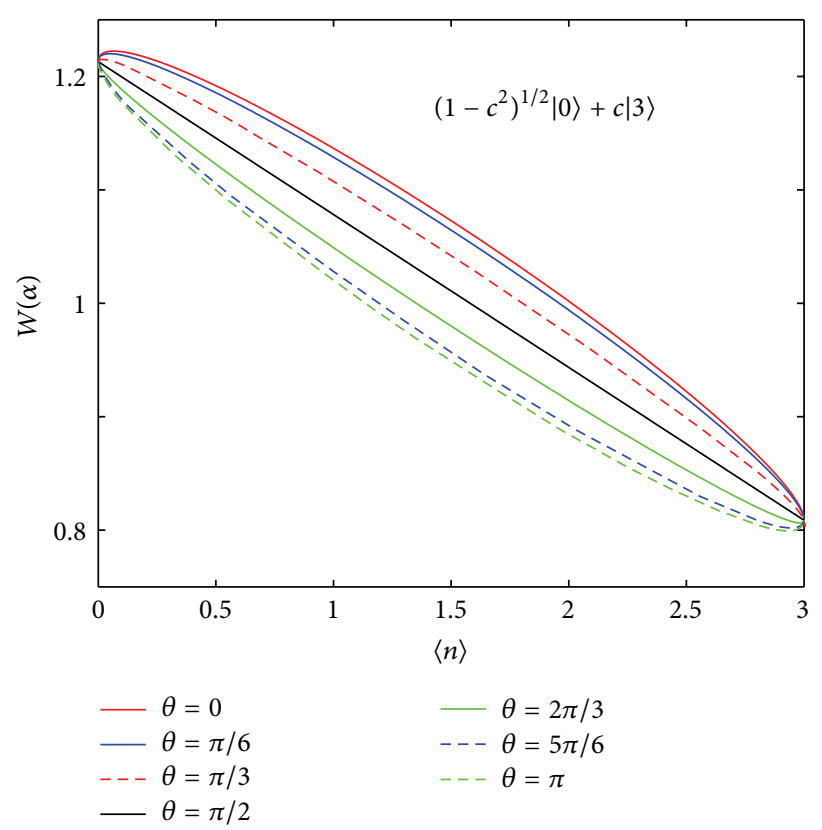

(b)

Figure 5: The Wigner functions for superposition of number eigenstates versus $\bar{n}$ : (a) for $\operatorname{Re}(\alpha)=0.2, \operatorname{Im}(\alpha)=0$ and $(\mathrm{b})$ for $\operatorname{Re}(\alpha)=0.5$, $\operatorname{Im}(\alpha)=0$.

Equations (5) are utilized to determine the value of $\langle n| m$, $2 \alpha\rangle$ in the above equations for $n, m=0,1$ as follows:

$$
\begin{aligned}
W(\alpha)=2 e^{-2|\alpha|^{2}}[( & \left.1-|c|^{2}\right) L_{0}^{0}\left(4|\alpha|^{2}\right) \\
& +\sqrt{1-c^{2}} c\left(e^{-i \theta} \alpha+e^{+i \theta} \alpha^{*}\right) L_{0}^{1}\left(4|\alpha|^{2}\right) \\
& \left.-|c|^{2} L_{1}^{0}\left(4|\alpha|^{2}\right)\right],
\end{aligned}
$$

where $\alpha=\operatorname{Re}(\alpha)+\operatorname{Im}(\alpha)$. By substituting (18) in (20) the Wigner function is obtained in terms of average photons number and the phase $\theta$ :

$$
\begin{aligned}
W(\alpha)= & 2 e^{-2|\alpha|^{2}} \\
& \times\left[(1-\bar{n}) L_{0}^{0}\left(4|\alpha|^{2}\right)+\sqrt{(1-\bar{n}) \bar{n}}\right. \\
& \left.\quad \times\left(e^{-i \theta} \alpha+e^{+i \theta} \alpha^{*}\right) L_{0}^{1}\left(4|\alpha|^{2}\right)-\bar{n} L_{1}^{0}\left(4|\alpha|^{2}\right)\right] .
\end{aligned}
$$

The value of Laguerre polynomials is calculated by

$$
L_{n}^{k}(x)=\frac{e^{x}}{n !} x^{-k} \frac{d^{n}}{d x^{n}}\left(e^{-x} x^{n+k}\right),
$$

for $x=4|\alpha|^{2}$ [24]. By (21) and (22) the Wigner distribution function for $\operatorname{Im}(\alpha)=0$ is obtained as

$$
W(\alpha)=2 e^{-2|\alpha|^{2}}\left[(1-2 \bar{n})+\sqrt{(1-\bar{n}) \bar{n}}|\alpha| \cos \theta+4 \bar{n}|\alpha|^{2}\right] .
$$

Clearly, the measurement of the Wigner function is more difficult for the large value of $\alpha$ because increasing the value of $\alpha$ gives a decrease in the value of the Wigner function. For $|\alpha| \ll 1,(23)$ reduces to $W(\alpha) \simeq 2 \exp \left(-2|\alpha|^{2}\right)(1-2 \bar{n})$, which is a linear in terms of $\bar{n}$ as shown in Figure 5. For this example our method gives us the value of the average number of photons even for $\alpha=0$.

3.2. Measuring the Average Number of Photons. Before running the experiment, the field of the cavity $C$ is set initially to be

$$
\left|\psi^{\prime \prime}\right\rangle=\widehat{D}(\alpha)\left|\psi^{\prime}\right\rangle=\sqrt{1-c^{2}}|0, \alpha\rangle+c|1, \alpha\rangle,
$$

by applying a coherent beam of light. The state of incoming atom is $|e\rangle$ which is resonantly (nonresonantly) interacted with the cavities $R_{1}$ and $R_{2}$ (with the cavity $C$ ) with a phase factor $\Omega_{R} t=\pi / 2$ (with a phase factor). Outgoing atom-field state is obtained as

$$
\begin{aligned}
|\psi\rangle= & \frac{1}{2}\left[\left(e^{-i \varphi(\widehat{n}+1)}-e^{i(\varphi \widehat{n}-\eta)}\right)|e\rangle\right. \\
& \left.+\left(e^{i(-\varphi((\hat{n}+1)+\eta)}+e^{i \varphi \widehat{n}}\right)|g\rangle\right] \\
& \times\left(\sqrt{1-c^{2}}|0, \alpha\rangle+c|1, \alpha\rangle\right) .
\end{aligned}
$$


Furthermore, the atom-field density matrix is obtained as

$$
\begin{aligned}
\widehat{\rho}_{\text {atom }+ \text { field }}=\frac{1}{4} & {\left[|e\rangle\langle e|\left(e^{-i \varphi(\widehat{n}+1)}-e^{i(\varphi \widehat{n}-\eta)}\right)\right.} \\
& \times \widehat{\rho}^{\prime}\left(e^{+i \varphi(\widehat{n}+1)}-e^{-i(\varphi \widehat{n}-\eta)}\right) \\
& +|g\rangle\langle g|\left(\left(e^{i(-\varphi(\widehat{n}+1)+\eta)}+e^{i \varphi \widehat{n}}\right)\right) \\
& \times \hat{\rho}^{\prime}\left(e^{i(-\varphi(\widehat{n}+1)+\eta)}+e^{-i \varphi \widehat{n}}\right) \\
& + \text { nondiagonal terms of atomic state }],
\end{aligned}
$$

where $\widehat{\rho}^{\prime}=\widehat{D}(\alpha) \widehat{\rho} \widehat{D}^{-1}(\alpha)$ and

$$
\begin{aligned}
\widehat{\rho}= & \left|\psi^{\prime}\right\rangle\left\langle\psi^{\prime}\left|=\left(1-|c|^{2}\right)\right| 0\right\rangle\langle 0| \\
& +\sqrt{1-c^{2}} c(|0\rangle\langle 1|+| 1\rangle\langle 0|)+|c|^{2}|1\rangle\langle 1| .
\end{aligned}
$$

The probability of finding the outgoing atom state in the states $|g\rangle$ or $|e\rangle$ is measured by an ionized detector. The difference between these probabilities $P_{e}-P_{g}$ is given by (15). By (15) and (27) and for $\varphi=-\eta=\pi / 2$, similar to (16), the measured Wigner function is obtained as

$$
\Delta P=P_{e}-P_{g}=\frac{1}{2} W(-\alpha) .
$$

The Wigner function, obtained with an experimental measurement of $\Delta P$, is compared to the value of displaced Wigner function, shown in Figure 5, to obtain the average of photons number $\bar{n}$. The development of this method to different superposition of two number states is straightforward. Figure 5 shows the Wigner function for a superposition of $|0\rangle$ and $|3\rangle$ number states which has many applications in the construction of a GHZ state [25]. As expected from (18), the average number of photons is independent of the phase $\theta$. Therefore by measuring the Wigner function in the point $\theta=0$ (or $\alpha=0)$ we can obtain the average number of photons and consequently the superposition coefficient $c$. Then by replacing the average number of photons in (23), the Wigner function is obtained in terms of the phase $\theta$. Measuring the Wigner function that is shown in Figure 6 leads us to obtain the phase $\theta$ for each superposition.

\section{Measuring the Squeezing Parameter}

4.1. Measuring the Squeezed Wigner Function. Usually the squeezed lights are produced by a nonlinear interaction of light and matter [26, 27]. Almeida et al. used two-photon interactions to produce the squeezed states [28]. If the field of the cavity $C$ is set to be in a displaced squeezed number state $|n, \xi, \alpha\rangle$, where $\xi=r e^{i \gamma}$ [6], we show that it is possible to determine the squeezing parameter $r$ by the proposed experimental setup. The states $|e\rangle$ and $|g\rangle$ of rubidium atoms resonantly interacted with the $R_{1}$ and $R_{2}$ cavities, where the phase $\Omega_{R} t$ is $\pi / 2$. Similar to Section 2, due to the nonresonance interaction of atom and field in the quantum cavity $C$, the state of atom is changed by a phase factor $\varphi$. The density operator for such a system is given by

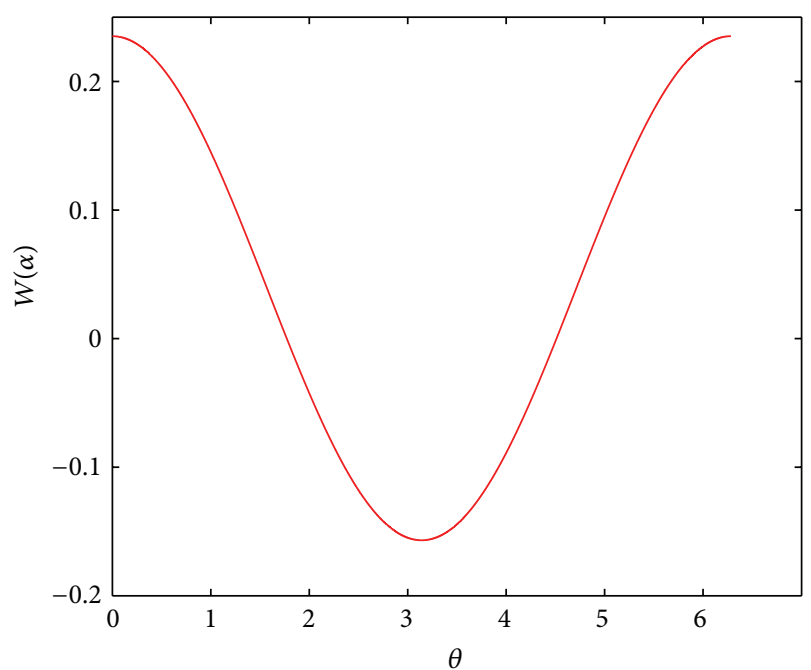

Figure 6: Plots of the Wigner function versus $\theta$ for $\operatorname{Re}(\alpha)=0.25$, $\operatorname{Im}(\alpha)=0$, and $\bar{n}=0.5$.

$\widehat{\rho}^{\prime \prime}=\widehat{D}(\alpha) \widehat{S}(\xi) \widehat{\rho} \widehat{S}^{-1}(\xi) \widehat{D}^{-1}(\alpha)$, where $\widehat{S}(\xi)=\exp \left((1 / 2) \xi^{*} \widehat{a}^{2}-\right.$ $\left.(1 / 2) \xi \hat{a}^{\dagger 2}\right)$ is a squeezing operator. Our main aim in this section is the measurement of the squeezed parameter $r$ at $\gamma=0$. The outgoing of state atom after passing through the cavities $R_{1}, C$, and $R_{2}$ is

$$
\begin{aligned}
|\psi\rangle=\frac{1}{2}\left[\left(e^{-i \varphi(\widehat{n}+1)}-e^{i(\varphi \widehat{n}-\eta)}\right)|e\rangle\right. \\
\left.\quad+\left(e^{i(-\varphi((\widehat{n}+1)+\eta)}+e^{i \varphi \widehat{n}}\right)|g\rangle\right]|n, \xi, \alpha\rangle .
\end{aligned}
$$

The density matrix of the atom-field system before any measurement is obtained as follows:

$$
\begin{aligned}
\widehat{\rho}_{\text {atom }+ \text { field }}= & \frac{1}{4}\left\{|e\rangle\langle e|\left(e^{-i \varphi(\widehat{n}+1)}-e^{i(\varphi \widehat{n}+\eta)}\right)\right. \\
& \times \widehat{\rho}^{\prime \prime}\left(e^{+i \varphi(\widehat{n}+1)}-e^{-i(\varphi \widehat{n}-\eta)}\right) \\
& +|g\rangle\langle g|\left(e^{i(-\varphi(\widehat{n}+1)+\eta)}+e^{i \varphi \widehat{n}}\right) \\
& \times \hat{\rho}^{\prime \prime}\left(e^{i(-\varphi(\widehat{n}+1)+\eta)}+e^{-i \varphi \widehat{n}}\right) \\
& + \text { nondiagonal terms of atomic state }\} .
\end{aligned}
$$

Detecting the atomic states with an ionized detector gives us the electromagnetic field state. Performing the experiment over and over the probabilities of finding atoms in $|g\rangle$ or $|e\rangle$ states are obtained. By (30) $\Delta P=P_{e}-P_{g}$ is obtained as

$$
\begin{aligned}
\Delta P & =-\operatorname{Re}\left\{e^{i(\varphi-\eta)} \operatorname{Tr}\left[\hat{\rho}^{\prime \prime} e^{2 i \varphi \widehat{a}^{\dagger} \widehat{a}}\right]\right\} \\
& =-\operatorname{Re}\left\{e^{i(\varphi-\eta)} \operatorname{Tr}\left[\widehat{D}(\alpha) \widehat{\rho}_{s} \widehat{D}^{-1}(\alpha) e^{2 i \varphi \widehat{a}^{\dagger} \widehat{a}}\right]\right\},
\end{aligned}
$$




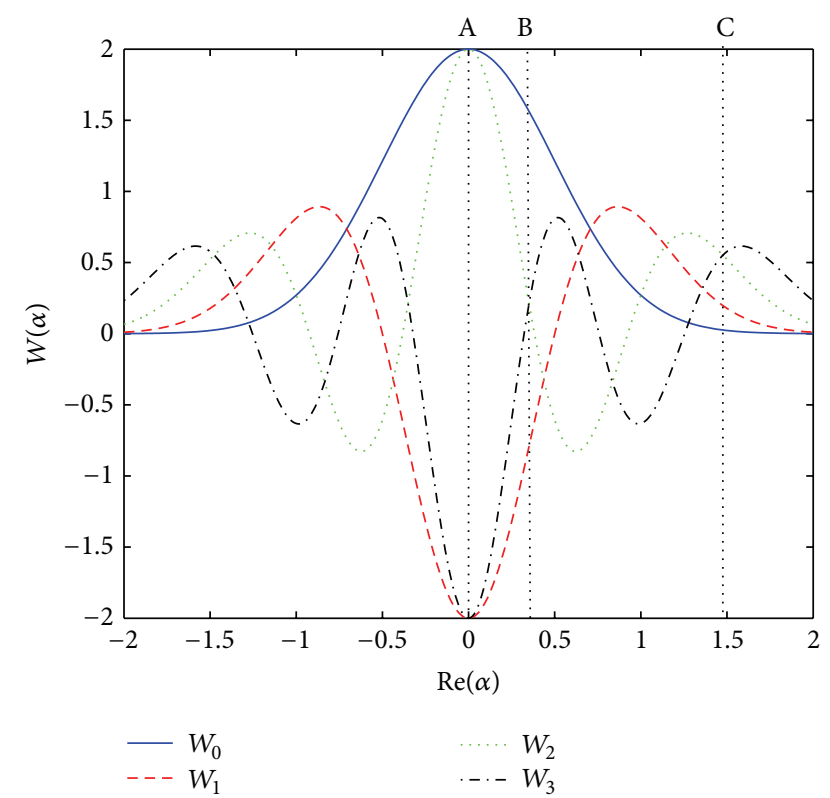

Figure 7: The plots of the Wigner function versus the real part of $\alpha$ in the regions between $\mathrm{A}$ and $\mathrm{B}$, between $\mathrm{B}$ and $\mathrm{C}$, and greater than $\mathrm{C}$. The point $\mathrm{A}$ is at the origin of phase space, $\mathrm{B}$ is the first intersection point of the curves, and $\mathrm{C}$ is the last interaction point of the curves.

where $\widehat{\rho}_{s}=\widehat{S}(\xi) \widehat{\rho} \widehat{S}^{-1}(\xi)$. It is very similar to (15) except $\widehat{\rho}$ which is replaced by $\hat{\rho}_{s}$. If the phase $\varphi=-\eta$ is set to $\pi / 2$, we have

$$
\begin{aligned}
\Delta P & =-\operatorname{Tr}\left[\widehat{D}(\alpha) \widehat{\rho}_{s} \widehat{D}^{-1}(\alpha) e^{i \pi \hat{a}^{\dagger} \hat{a}}\right] \\
& =-\operatorname{Tr}\left[\widehat{D}(\alpha) \widehat{S}(\xi) \hat{\rho} \widehat{S}^{-1}(\xi) \widehat{D}^{-1}(\alpha) e^{i \pi \widehat{a}^{\dagger} \widehat{a}}\right] .
\end{aligned}
$$

Clearly, the above equation is proportional to the squeezed displaced Wigner function:

$$
\Delta P=\frac{1}{2} W_{\xi}(-\alpha) .
$$

Equation (33) shows a relation between the experimental value $\Delta P$ and the squeezed displaced Wigner function (or squeezing parameter $r$ ), which is given theoretically. Therefore the squeezing parameter $r$ is obtained by the measurement of $\Delta P$.

4.2. Calculating the Squeezed Wigner Function. As shown in Section 2, the Wigner function is written in terms of the Laguerre polynomials for a number state. It is also possible to write the Wigner function for a squeezed number state in terms of the Laguerre polynomials. For a squeezed number state, the Wigner function can be obtained by replacing $e^{r} q$ and $e^{-r} p$ with $q$ and $p$, for the Wigner function at $\gamma=0^{\circ}$ [27], respectively. Therefore the Wigner function of a squeezed displaced number state is given by

$$
\begin{aligned}
W_{n r}(\alpha)= & 2(-1)^{n}\left\{L_{n}\left(4\left(e^{2 r}(\operatorname{Re}(\alpha))^{2}+e^{-2 r}(\operatorname{Im}(\alpha))^{2}\right)\right)\right\} \\
& \times \exp \left(-2\left(e^{2 r}(\operatorname{Re}(\alpha))^{2}+e^{-2 r}(\operatorname{Im}(\alpha))^{2}\right)\right) .
\end{aligned}
$$

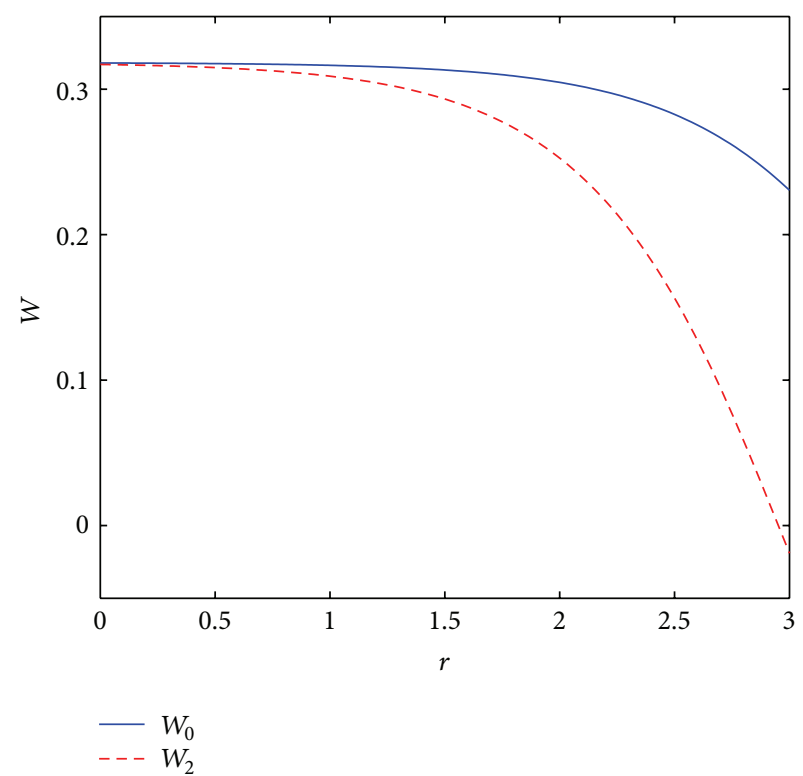

(a)

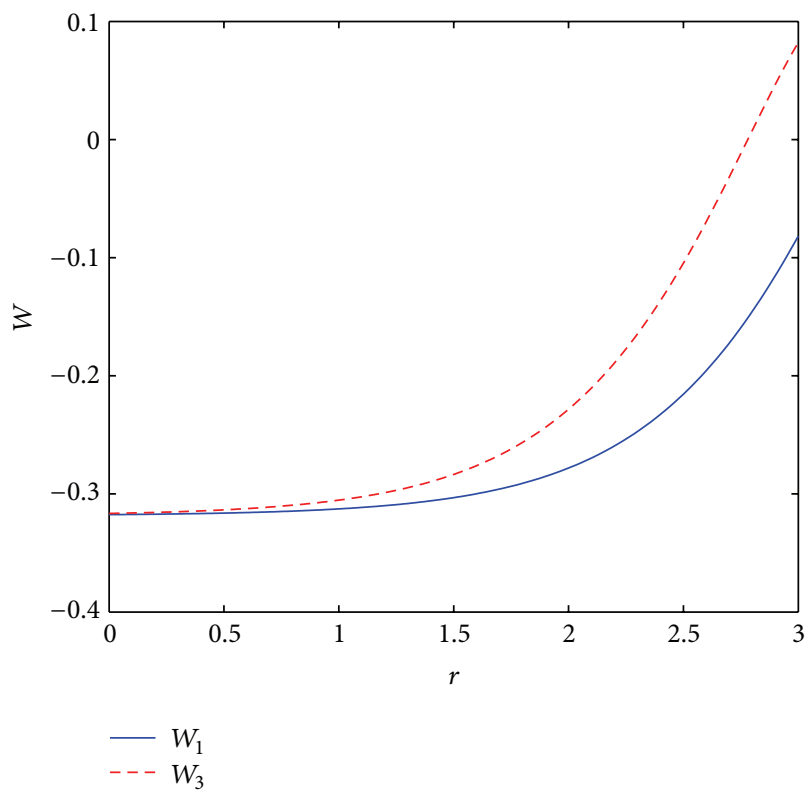

(b)

FIGURE 8: Plots of the Wigner function versus $r$ for $\operatorname{Re}(\alpha)=0.02$ and $\operatorname{Im}(\alpha)=0$. As illustrated, these plots are more suitable to measure the greater values of $r$ because the difference between the Wigner functions is larger than small value of $r$.

In (34) the squeezed Wigner function is obtained by replacing $e^{r} \operatorname{Re}(\alpha)$ and $e^{-r} \operatorname{Im}(\alpha)$ with $\operatorname{Re}(\alpha)$ and $\operatorname{Im}(\alpha)$. For a number state, the Wigner functions versus the squeezing parameter are plotted for 0 to 3 number states versus $\operatorname{Re}(\alpha)$ in Figure 7 . In order to set $\alpha$ in the best point for the measurement of the squeezing parameter $r$, we partitioned the horizontal axis into three domains.

(1) The A-B domain is relevant to small $\alpha$. This domain is started from the origin to the first intersection point 


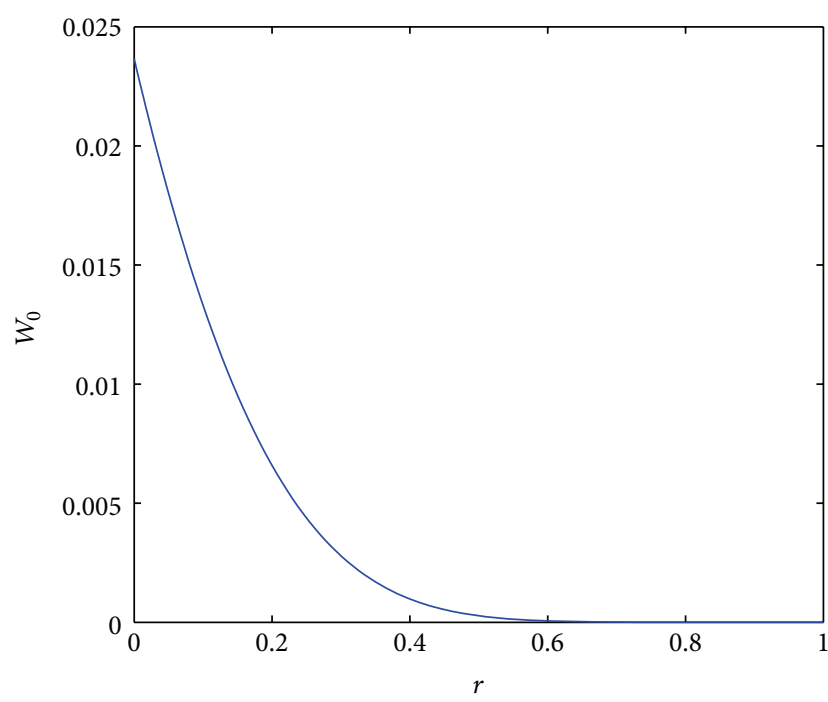

(a)

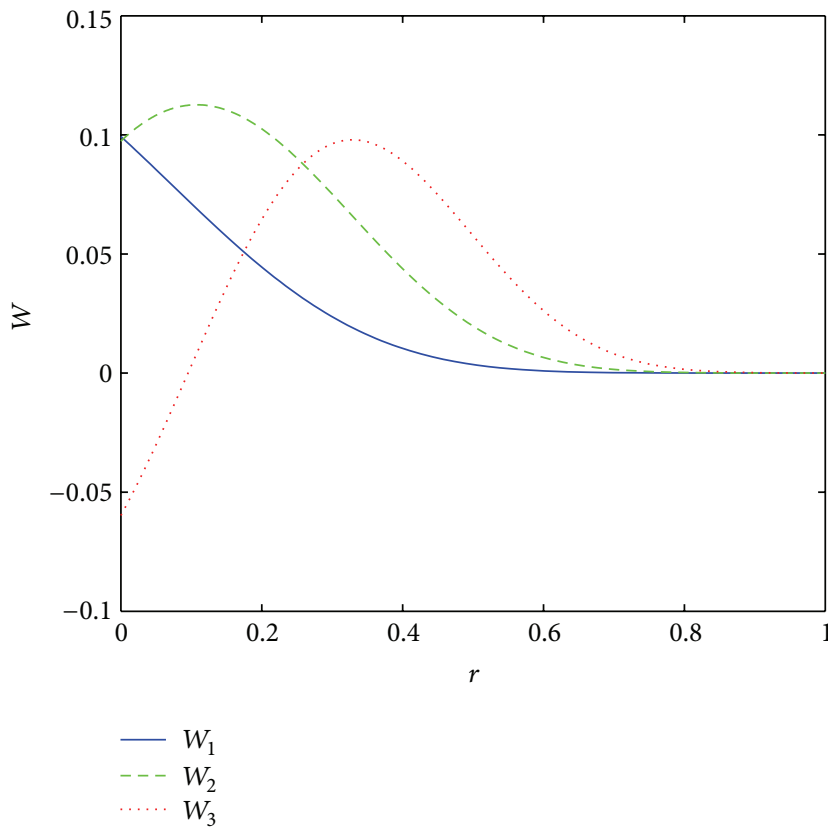

(b)

FIGURE 9: Plots of the Wigner functions for number states versus $r$, for $\operatorname{Re}(\alpha)=1.14, \operatorname{Im}(\alpha)=0$.

of the curves. Near the middle, the difference between the Wigner functions is almost larger than that of the other points. So selecting $\alpha$ in the middle of this domain is a more suitable selection for the measuring of the squeezing parameter $r$. In Figure 8 the Wigner functions are plotted versus $r$ for 0 to 3 photon number in the domain A-B. It has been shown that for a single value of a Wigner function and a specific number state, the value of squeezing parameter is unique. The sensitivity of Wigner function is higher for larger $r$. We conclude that smaller values of $\alpha$ are more suitable for measuring larger squeezing parameters.

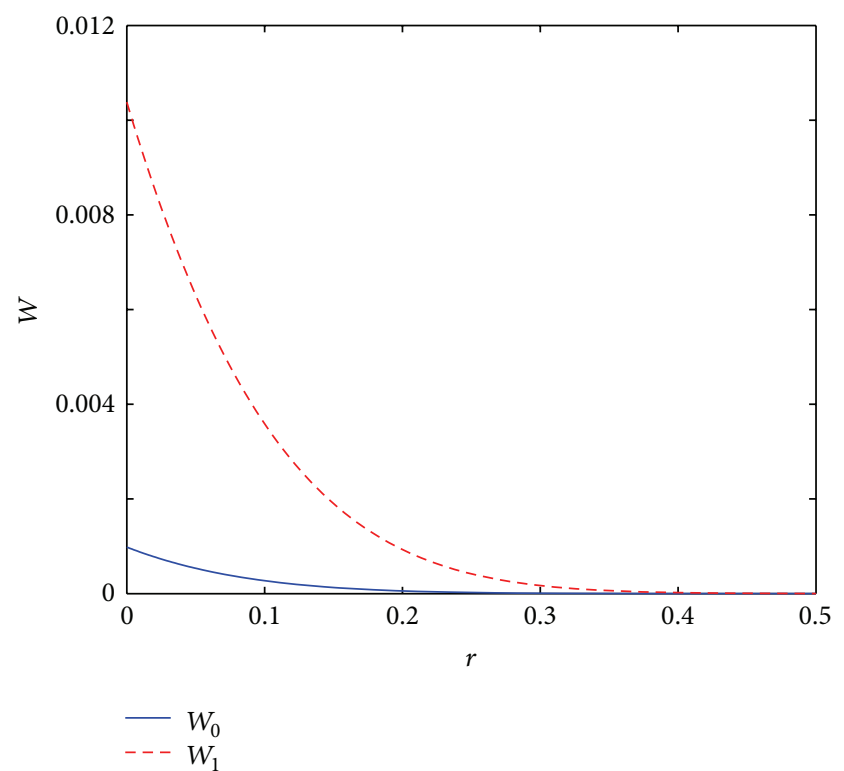

(a)

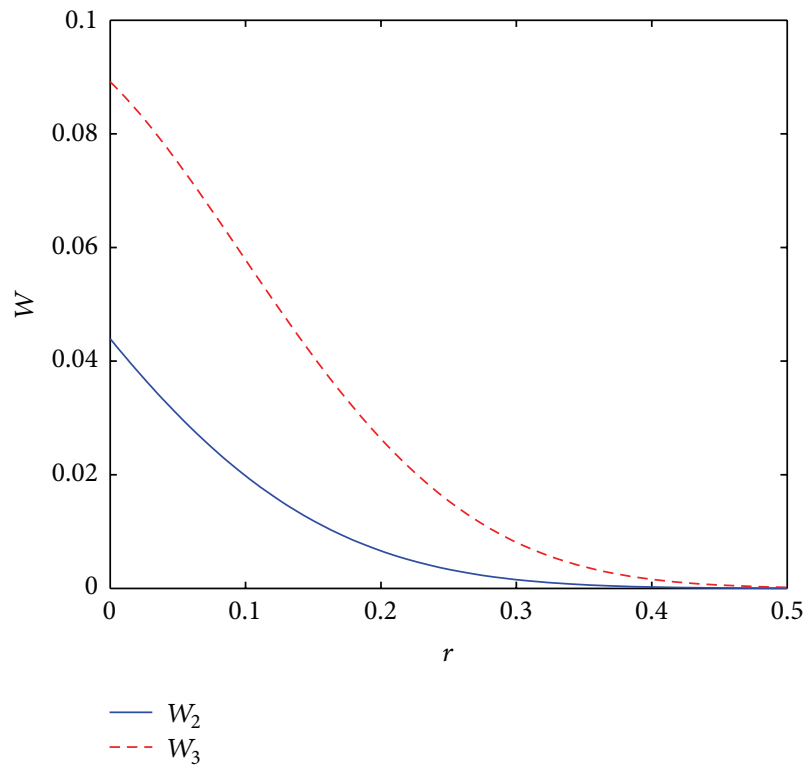

(b)

FIGURE 10: Plot of the Wigner function versus $r$ in the points $\operatorname{Re}(\alpha)=$ 1.7 and $\operatorname{Im}(\alpha)=0$ for the interval $0 \leq r \leq 0.5$.

(2) The B-C domain is relevant to the middle value of $\alpha$. This domain is from the first intersection point of the two curves and extends out till the last intersection. Usually selection of $\alpha$ in this domain is not suitable at all, since for a single value of a Wigner function the value of squeezing parameter is not unique. Figure 9 shows this nonuniqueness of the squeezing parameter for any Wigner function.

(3) The domain greater than $C$ is relevant to the bigger values of $\alpha$. In this domain squeezing parameter is unique for any Wigner function. It should be noticed that for bigger values of squeezing parameter the 
Wigner functions are very small. Figure 10 illustrates the Wigner functions versus squeezing parameter $r$ for $\operatorname{Re}(\alpha)=1.7$ and $\operatorname{Im}(\alpha)=0$. It decreases for bigger $\alpha$ and the sensitivity of the Wigner functions is higher for smaller $r$. Therefore, the domain $C$ is more suitable for the measurement of small $r$. Here, the value of the Wigner function for bigger $\alpha$ is very small, so it is not a suitable measurable value. Therefore, we choose $\operatorname{Re}(\alpha)$ to be not very far from the point $C$.

\section{Conclusion}

In this paper we use measurement of the displaced Wigner function for the measuring of photons number in a quantum cavity. In this method a two-level Rydberg Rubidium atom is used to make a nondemolition measurement of the average number of photons in cavities. Detection of the atom states gives us $P_{e}$ and $P_{g}$. It is shown that the difference between $P_{e}$ and $P_{g}$ gives us the displaced Wigner function and further gives us the average number of photons in the cavity in a nondemolition measurement method. This setup has also been used to the nondemolition measurement of the squeezing parameter $r$ in the field of quantum cavity. One may measure the squeezing parameter by measuring the displaced squeezed Wigner function. In order to obtain either a unique $r$ for each measured Wigner function or to increase the sensitivity of our measurements, the value of displacement should be set. We find that for larger values of squeezing parameter $r$ the displacement should be small and for smaller values of squeezing parameter $r$ the displacement should be large.

\section{References}

[1] E. P. Wigner, "On the quantum correction for thermodynamic equilibrium," Physical Review, vol. 40, no. 5, pp. 749-759, 1932.

[2] A. G. Baker Jr., "Formulation of quantum mechanics based on the quasi-probability distribution induced on phase space," Physical Review, vol. 109, no. 6, pp. 2198-2206, 1958.

[3] K. C. Zachos, B. D. Fairlie, and L. T. Curtright, Quantum Mechanics in Phase Space, World Scientific, Singapore, 2005.

[4] R. J. Glauber, "The quantum theory of optical coherence," Physical Review, vol. 130, no. 6, pp. 2529-2539, 1963.

[5] R. J. Glauber, "Coherent and incoherent states of the radiation field," Physical Review, vol. 131, no. 6, pp. 2766-2788, 1963.

[6] M. O. Scully and M. S. Zubairy, Quantum Optics, Cambridge University Press, Cambridge, UK, 1997.

[7] P. Deuar and P. D. Drummond, "First-principles quantum dynamics in interacting Bose gases. I. The positive P representation," Journal of Physics A, vol. 39, no. 5, article 1163, 2006.

[8] P. D. Drummond, P. Deuar, and K. V. Kheruntsyan, "Canonical bose gas simulations with stochastic gauges," Physical Review Letters, vol. 92, no. 4, Article ID 040405, 4 pages, 2004.

[9] G. M. Benedict and A. Czirják, "Wigner functions, squeezing properties, and slow decoherence of a mesoscopic superposition of two-level atoms," Physical Review A, vol. 60, no. 5, pp. 4034-4044, 1999.

[10] P. Faldi, A. Czirják, B. Molnár, and G. M. Benedict, "Formation of schrödinger-cat states in the morse potential: wigner function picture," Optics Express, vol. 10, no. 8, pp. 376-381, 2002.
[11] P. Sadeghi, S. Khademi, and S. Nasiri, "Nonclassicality indicator for the real phase-space distribution functions," Physical Review A, vol. 82, no. 1, Article ID 012102, 8 pages, 2010.

[12] D. Leibfried, D. M. Meekhof, B. E. King, C. Monroe, W. M. Itano, and D. J. Wineland, "Experimental determination of the motional quantum state of a trapped atom," Physical Review Letters, vol. 77, no. 21, pp. 4281-4285, 1996.

[13] K. Banaszek and K. Wódkiewicz, "Direct probing of quantum phase space by photon counting," Physical Review Letters, vol. 76, no. 23, pp. 4344-4347, 1996.

[14] S. Wallentowitz and W. Vogel, "Unbalanced homodyning for quantum state measurements," Physical Review A, vol. 53, no. 6, pp. 4528-4533, 1996.

[15] C. Guerlin, J. Bernu, S. Deléglise et al., "Progressive fieldstate collapse and quantum non-demolition photon counting," Nature, vol. 448, pp. 889-893, 2007.

[16] S. Deléglise, I. Dotsenko, C. Sayrin et al., "Reconstruction of non-classical cavity field states with snapshots of their decoherence," Nature, vol. 455, no. 7212, pp. 510-514, 2008.

[17] P. Bertet, A. Auffeves, P. Maioli et al., "Direct measurement of the wigner function of a one-photon fock state in a cavity," Physical Review Letters, vol. 89, no. 20, Article ID 200402, 4 pages, 2002.

[18] L. G. Lutterbach and L. Davidovich, "Method for direct measurement of the wigner function in cavity QED and ion traps," Physical Review Letters, vol. 78, no. 13, pp. 2547-2550, 1997.

[19] L. G. Lutterbach and L. Davidovich, "Non-classical states of the electromagnetic field in cavity QED," Optics Express, vol. 3, no. 4, pp. 147-153, 1998.

[20] G. Nogues, A. Rauschenbeutel, S. Osnaghi et al., "Measurement of a negative value for the wigner function of radiation," Physical Review A, vol. 62, no. 5, Article ID 054101, 4 pages, 2000.

[21] K. E. Cahill and R. J. Glauber, "Density operators and quasiprobability distributions," Physical Review, vol. 177, no. 5, pp. 1882-1902, 1969.

[22] M. G. Benedict and A. Czirjak, "Generalized parity and quasiprobability density functions," Journal of Physics A, vol. 28, no. 16, article 4599, 1995.

[23] C. Gerry and P. Knight, Introductory Quantum Optics, Cambridge University Press, Cambridge, UK, 2004.

[24] G. B. Arfken and H. J. Weber, Mathematical Methods for Physicists, Elseveir Academic Press, Burlington, Mass, USA, 2005.

[25] J. I. Cirac and P. Zoller, "Preparation of macroscopic superpositions in many-atom systems," Physical Review A, vol. 50, no. 4, pp. R2799-R2802, 1994.

[26] R. E. Slusher, L. W. Hollberg, B. Yurke, J. C. Mertz, and J. F. Valley, "Observation of squeezed states generated by four-wave mixing in an optical cavity," Physical Review Letters, vol. 55, no. 22, pp. 2409-2412, 1985.

[27] L. Ulf, Measuring the Quantum State of Light, Cambridge University Press, Cambridge, UK, 1997.

[28] N. G. de Almeida, R. M. Serra, C. J. Villas-Bõas, and M. H. Y. Moussa, "Engineering squeezed states in high-Q cavities," Physical Review A, vol. 69, no. 3, Article ID 035802, 4 pages, 2004. 

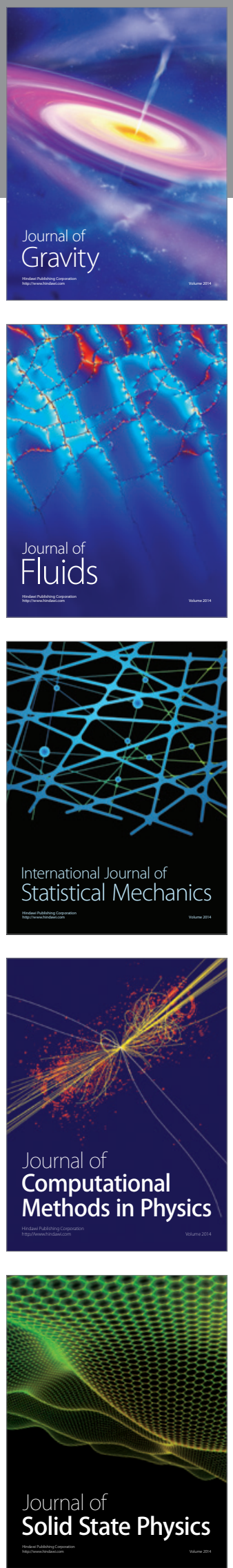

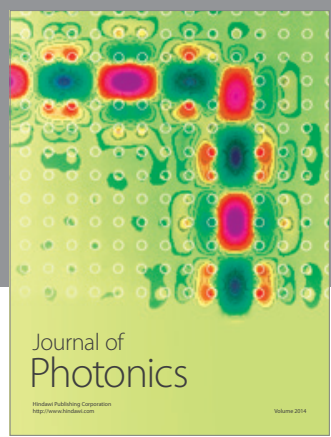

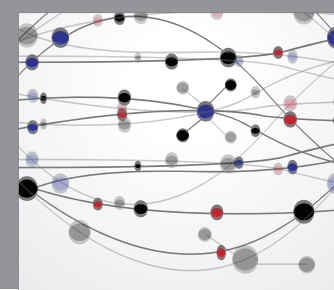

The Scientific World Journal

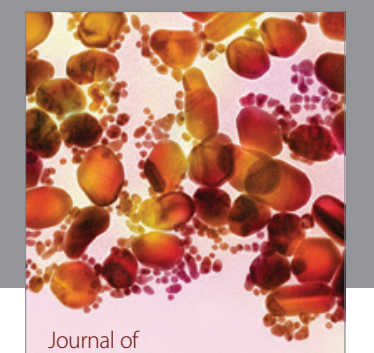

Soft Matter
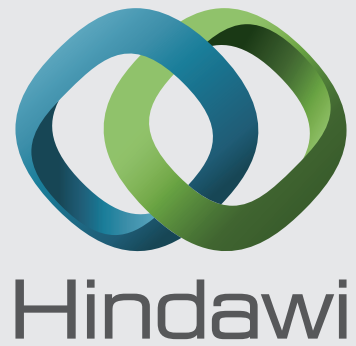

Submit your manuscripts at

http://www.hindawi.com
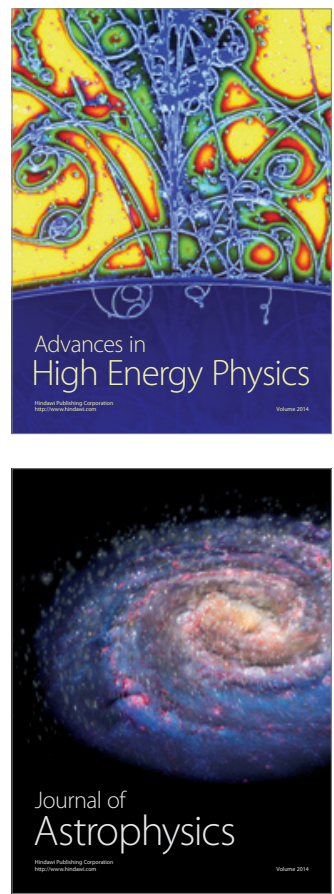
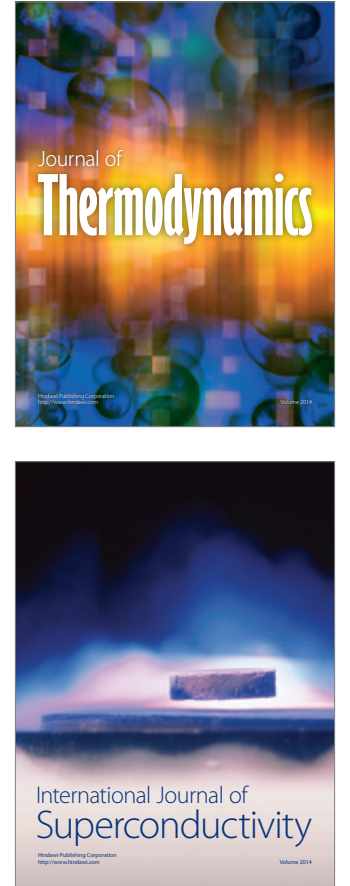
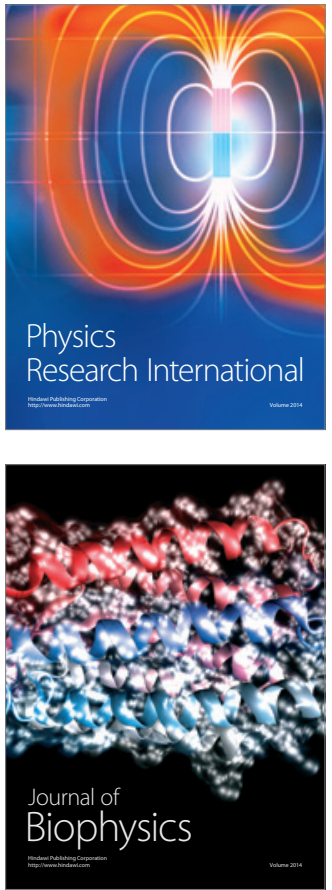
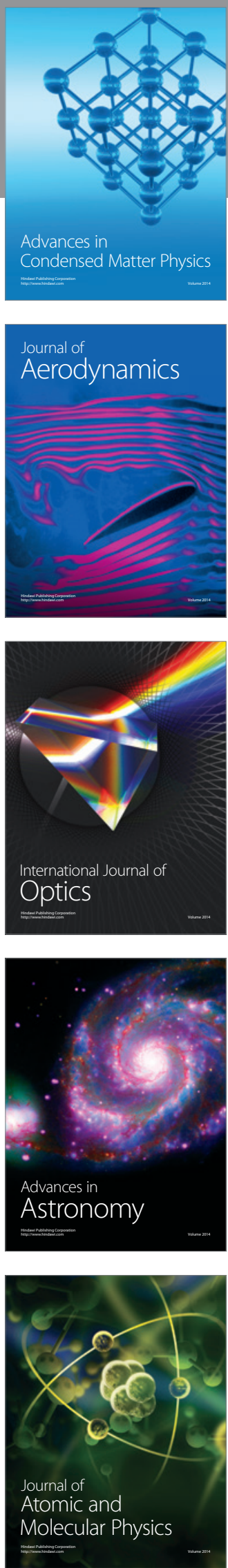